\title{
1.1 Distributed Knowledge Management in Dynamic Environments
}

\author{
Hagen Langer, Jan D. Gehrke, Otthein Herzog \\ TZI - Center for Computing Technologies, Universität Bremen, \\ Am Fallturm 1, 28359 Bremen, Germany
}

\subsubsection{Introduction}

Logistic processes are inherently dynamic and hence require the ability to plan and re-plan in complex situations, under rigid time constraints, and in light of uncertain, incomplete, and false information. Standard scenarios of logistic processes typically have been modeled on the basis of static graphtheoretic representations. The well-known traveling salesman problem (TSP), the vehicle routing problem (VRP), or the pickup \& delivery problem (PDP) reduce the complex task of transportation to a route optimization problem. They neglect both the important role of knowledge and communication in real-world logistic processes (cf. (Hult et al. 2003)) and the fact that relevant parameters, e.g., traffic flow, incoming orders, etc. change over time.

In this paper we will describe an approach to the agent-based modeling of logistic processes which makes use of an explicit knowledge management system and hence enables agents to fulfill complex logistic tasks in dynamic environments.

This paper is organized as follows. We introduce agents as basic components of our framework in Sect. and discuss agent-based approaches to logistics (). Section presents our approach to distributed knowledge management for multiagent systems. We discuss agent roles, decision parameters, and an interaction protocol for the two most important knowledge management roles of our framework. In Sect. we summarize the main conclusions of this work. 


\subsubsection{Intelligent Agents}

Agents are currently one of the most prominent paradigms for creating autonomous software systems. A broad variety of agent architectures have been proposed in the past. One extreme in the spectrum of agent architectures are reactive agents which are not necessarily much more autonomous in their decision-making than standard software components, but share other important properties with prototypical agents (e.g., the existence of sensors and actuators). The other extreme is established by cognitive agents, which mimic our assumptions on human cognitive processes as close as possible. Cognitive agents are often implemented as BDI agents (belief, desire, and intention), cf. (Rao and Georgeff 1991). BDI agents possess an autonomous knowledge base (the beliefs) and this knowledge base is modified whenever the agent interacts with its environment or when the agent updates its knowledge base by inferring new knowledge from its existing background knowledge. In the simplest case, this interaction means that the agent receives percepts via its sensors. The behavior of a BDI agent is also determined by its desires and its intentions. The desires are long-term goals which, together with the beliefs, determine the agent's intended actions. The beliefs and actions of a BDI agent depend not only on the agent's environment but also on the agent's existing knowledge base and its desires.

\subsubsection{Agent-based Logistics}

Previous research on applying multiagent systems in the logistics domain has put a strong emphasis on price negotiations and auctions. In these approaches the inter-agent communication is often reduced to bidding (cf., e.g., (Zhengping et al. 2001)), or the internal structure of an agent is defined by a set of equations (e.g., (Bos et al. 1999)). Scholz et al. (2004) apply MAS to shop floor logistics in a dynamic production scenario. It aims at a flexible and optimal scheduling of production plans in a heterogeneous shop floor environment. Hofmann et al. (1999) aim at replacing conventional tracking and tracing in the logistics domain based on sending (i.e., pushing) EDIFACT messages by an agent-based pull mechanism. Smirnov et al. (2003) present a prototype of a multi-agent community implementation and a constraint-based protocol designed for the agents' negotiation in a collaborative environment. Most of the previous approaches to multiagent-system-based logistics, however, employ simplified models of logistic processes which do not involve any explicit knowledge management. 
Our approach is based on a system of autonomous agents which represent logistic entities. Besides its primary logistic functionality, each agent can adopt a role as part of a distributed knowledge management system.

Our framework makes the following assumptions about real-world logistic scenarios

- Real-world logistic scenarios are never static, but highly dynamic.

- Agents involved in logistic processes have to plan and act on the basis of incomplete, uncertain, and rapidly changing knowledge.

- Optimal decision making under the circumstances sketched above presupposes an appropriate knowledge management framework.

- Knowledge is a valuable resource and can also be a tradable good.

We can envision a scenario in which agents are used to represent realworld entities such as trucks and containers, abstract objects such as weather or traffic services, or even human decision makers, such as a ramp agent at a loading dock. We believe this kind of autonomous, decentralized decision-making can help make the operational processes more efficient, cost-effective, and allow the participating enterprise to stay competitive. It is also a major improvement over traditional centralized approaches in which individual agents are ill-equipped to deal quickly with sudden events since control usually resides with the entities that are removed from the scene of the event and thus have only delayed access to the relevant information. In addition, agents must be able to negotiate, form coalitions, and thrive in the presence of competition, for example, for customers (orders) or resources, and are also subject to unpredictable changes in their environment.

In contrast to standard approaches to the computational modeling of transportation processes, we do not presuppose that there is a central omniscient unit which plans, coordinates, and controls the activities of logistic entities (e.g., vehicles, depots). We, on the contrary, assume that these logistic entities are autonomous and control themselves. This setting requires that there is a robust and flexible knowledge management system which is able to provide the necessary knowledge for each agent.

\subsubsection{Knowledge Management based on Roles and Parameters}

Agent-based knowledge management has been studied under different assumptions, but the main focus of previous research has been on single agents, as opposed to multiagent systems (MAS), which we employ, and

1 By standard approaches we mean settings such as the well-known traveling salesman problem (TSP), the vehicle routing problem (VRP), or the pickup delivery problem (PDP). 
on knowledge management by agents for human users, as opposed to our approach which is not only by agents but moreover for agents. Another important difference between our framework and previous agent-based knowledge management systems is that we do not presuppose a one-to-one mapping between agents, on the one hand, and knowledge management functions, on the other.

Three main components are ingredients of our framework: agents, knowledge, and roles. Agents represent process owners (e.g., decision makers) or real-world entities in the logistics domain (e.g., cargo transport centers, vehicles, transport containers, or even single packages).

In addition, an agent has specific properties (e.g., speed, weight, enterprise affiliation), capabilities (e.g., transportation or storage capabilities, or sensors for measuring humidity), desires (e.g., minimizing delay of a shipment or maximizing the utilization ratio), and intentions (i.e., tactical plans). The set of beliefs forms an agent's knowledge base and is associated with specific inferential capabilities.

We envision that these agents, which must act in a rational fashion, can be implemented as goal-oriented agents following the BDI (belief, desire, intention) approach as discussed above. The BDI approach is well suited for this purpose since it provides the appropriate concepts and structures for representing our agents. For example, the strategic layer of agents may be modeled within the desires, operational aspects within beliefs, and tactical features within intentions or plans. Furthermore, the BDI approach attempts to closely mimic human decision-making (Bratman 1987) and represents one of the dominant approaches for modeling intelligent behavior within the agent research community (d'Inverno et al. 2004). For a comprehensive discussion of the applicability of BDI to represent rational, autonomous agents see also (Timm 2004).

The second component of our framework provides knowledge management functionalities including knowledge representation, storage, and manipulation. In our framework, the terminological domain knowledge is organized in associated ontologies for transportation and production logistics which include, e.g., a representation of the transportation network as an annotated graph, together with a two-dimensional map-like representation (similar to geographic information systems) enabling spatial reasoning (e.g., inferring properties of proper sub-regions using a part-of relation), the basic types of agent and their properties (e.g., for a vehicle, its average and maximum speed, the types of routes in the network it can use, its load capacity, and its corporate affiliation), and the properties of 'inactive' objects, such as highways, traffic hubs, depots, etc.

The visibility of the ontology is determined by an agent's predefined tasks and capabilities. For example, in contrast to a shipment agent, an 
agent representing a navigation system must have complete access to all relevant details of the transportation network part of an ontology.

Knowledge management enables agents to request new or missing knowledge, or update existing knowledge. Intuitively, our approach is similar to peer-to-peer knowledge management. Agents have the ability to form dynamic knowledge networks and to share knowledge. Hereby knowledge management becomes a secondary task orthogonal to the primary logistic tasks.

The third component of our framework integrates the multiagent approach with knowledge management functionalities using roles. Examples of these roles are knowledge acquisition, brokerage, and processing. Depending on their capabilities and tasks in the logistics domain, agents may assume any one of these roles, which may change over time. For example, an agent representing a ship may assume the role of a knowledge provider reporting weather information to other ships. At a different point in time, the same agent may also assume the role of a knowledge consumer requesting information about its cargo and destination from a dock agent after loading is complete. Communication among agents is implemented by the already existing agent communication infrastructure.

In contrast to conventional knowledge management systems, our approach is inherently distributed. In particular, it focuses on knowledge management performed by agents and for agents as decision makers in logistic processes. Nevertheless, humans remain an important factor because they need the capability to monitor the logistic processes and the agents therein.

As a prerequisite to apply our framework we are tacitly assuming the existence of standard information technologies to provide the proper support such as networking, document storage, retrieval, metadata annotation, etc. Despite potentially existing connections by corporate affiliation, we do not presuppose initial structures in the knowledge management network. In contrary, as argued above, we emphasize the necessity of dynamic situation- and location-dependent interactions. In a sense, the structure of the knowledge management system emerges from the interaction of agents by virtue of implementing specific roles autonomously and in dynamic change.

Knowledge management as it is proposed in this framework is one key enabling factor to the envisioned autonomy of logistic processes. Autonomous entities need to make decisions based on a technically implemented decision theoretic process. In order to achieve this they not only need knowledge about their environment, but also have to assess possible future states of this environment and judge alternative options. In (Lorenz et al. 2005) we propose a mechanism for assessing the risk associated with 
an option based on knowledge the agent has about its current environment. This risk management is very closely related to knowledge management (cf. (Bemeleit et al. 2006) in this volume). On the one hand it can trigger the acquisition of additional knowledge. On the other hand it may be necessary to evaluate the risk linked with a KM decision, e.g., giving away certain information or asking an expensive but reliable source instead of a free but inaccurate one.

It is important to note that distributed knowledge management is restricted by various sociological and technological boundaries. For example, on a sociological level, agents may represent competing enterprises, which may lead to inconsistent or even incompatible desires. In addition, there is the important issue of trust. Low trust levels could prevent agents to assume certain roles (e.g., that of a knowledge broker or provider). High trust levels strengthen the connections between certain agents, causing an increase in traffic over time. As far as technological boundaries are concerned, the presence of embedded computational entities, which are partially moving in the physical world, leads to hard restrictions on network availability and computational power.

\section{Agent Roles}

The agent-oriented approach, which advocates decomposing problems in terms of autonomous agents that can engage in flexible, high-level interactions (Jennings 2000), employs a multitude of agents to solve the knowledge management problem. In our approach to distributed knowledge management the agents have a special primary task, e.g., self-organization of a logistic entity. Managing and sharing knowledge becomes an optional secondary capability orthogonal to their primary logistic task. Thus, in contrast to previous approaches to agent-based knowledge management (van Elst et al. 2004a), there is no one-to-one correspondence between agents and knowledge management functions, such as providing knowledge or brokering knowledge.

In order to cope with this system characteristic we map knowledge management functions onto agent roles. Herrmann et al. (2004) report on a number of case studies which show that in sociologically inspired systems (in that case a collaborative learning environment) users "attempted to take different roles and tried to change their roles dynamically in being able to structure their communication." They give an overview on the application of sociological role concepts in computer supported collaboration and state a need for role development in computer-supported knowledge management. In a sociologically inspired computer system, e.g., a MAS, it seems therefore straightforward to apply the role metaphor from computer-sup- 
ported $\mathrm{KM}$ for humans to $\mathrm{KM}$ for agents. This is especially true as human agents are explicitly included in the overall concept.

Within our framework a knowledge management role includes certain reasoning capabilities, a visibility function on an agent's beliefs, a deliberation pattern (i.e., a plan how to accomplish the KM task), and a communication behavior with interacting roles. The aim of $\mathrm{KM}$ roles is to provide a formal description of knowledge management tasks that eases the development of agents and reduces the computational complexity by means of a minimum set of processed knowledge and applied reasoning capabilities. One agent can assume different roles and may switch them over time. The minimum role model includes the roles of a provider offering information and a consumer being in need of information. The next extension would be a broker mediating between the two (van Elst et al. 2004b). Taking the agent-based approach, our claim to fully automate knowledge management raises new reasoning demands especially on the brokering and maintenance of knowledge, which have not been addressed so far. For example, in classical KM approaches, knowledge brokering and maintenance are performed by human actors (cf. (Maurer 2003)). We propose an extended role model that incorporates all knowledge management functions we identified as needed for autonomous logistic processes.

We distinguish internal and external roles. The latter ones are interactive and presuppose at least two involved agents, the former ones do not require inter-agent communication, but refer to intra-agent processes. Both types of roles are independent from the primary logistic task of an agent and define a complex behavior which results in a modification of an agent's knowledge base.

Figure 1.1 depicts a conceptual overview of the most important external roles in our framework together with the corresponding communication acts. Figure 1.2 shows the internal roles' operations with respect to an agent's knowledge base. We briefly describe the resulting role set and the respective tasks in our proposed framework: 


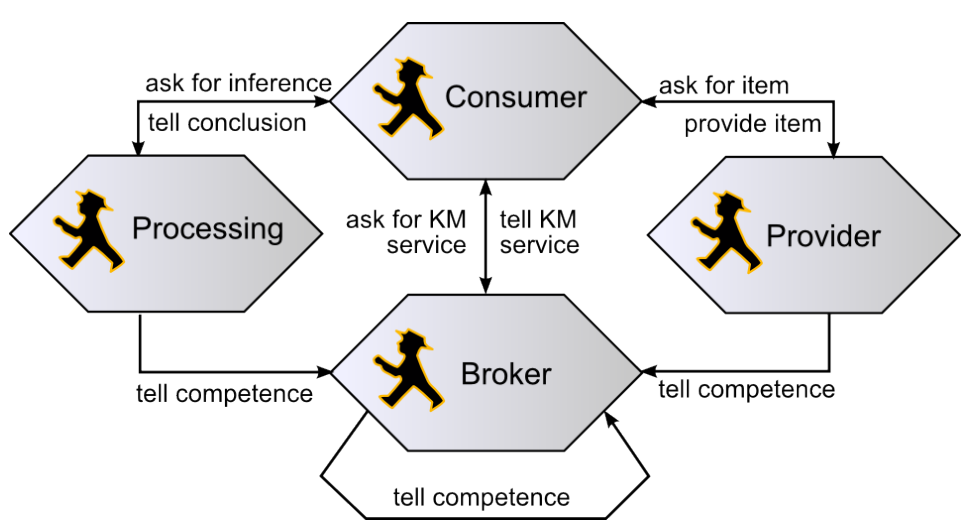

Fig. 1.1 External roles

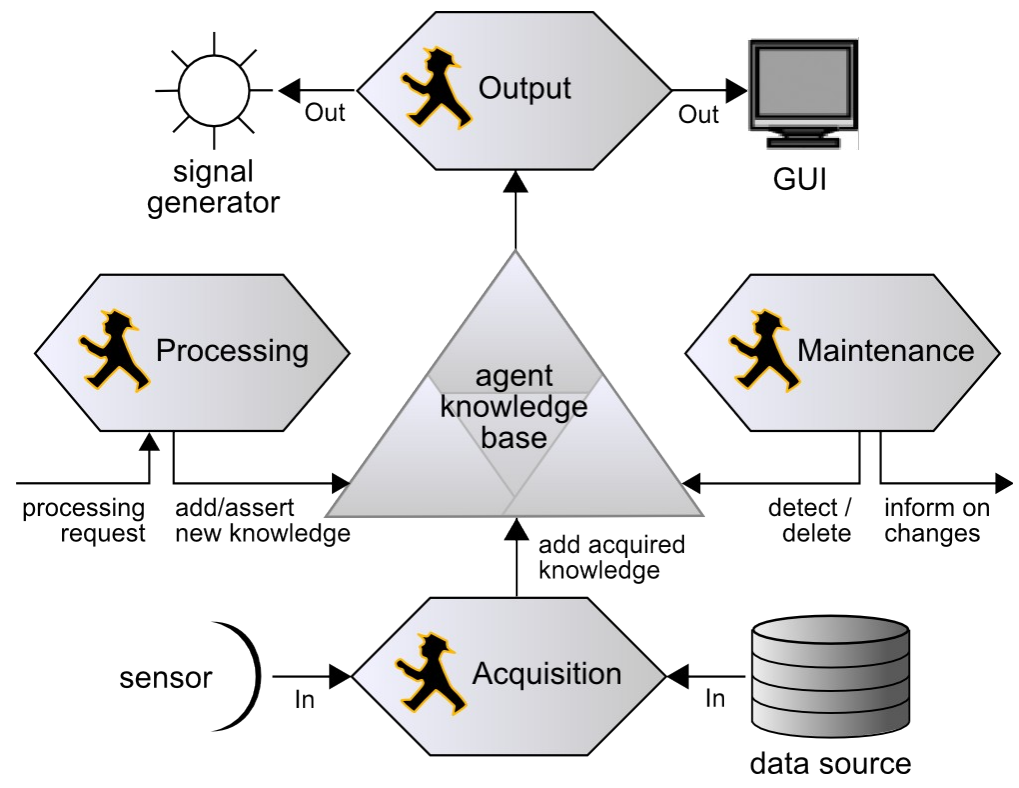

Fig. 1.2 Internal roles

Knowledge Consumer: An agent acts as a knowledge consumer the moment it discovers a lack of its own local knowledge. Which knowledge it considers to be most important depends both on the agent's current knowledge base and its environment, and will be explained in more detail in Sect. . In order to determine the most appropriate knowledge source the agent uses its meta-knowledge on its inferential abilities, own sensors, 
available data sources, and provider agents. If the agent decides to ask another agent and its meta-knowledge on adequate services is considered insufficient the agent may consult a broker (see below) as for who would be able to provide the needed service. Successful direct transfers with providers will strengthen the relationship to them and decrease the necessity to use brokers.

Knowledge Provider: An agent assuming the knowledge provider role in a knowledge transfer process provides parts of its internal knowledge repository either on demand or as part of some pro-active behavior. To be able to provide knowledge pro-actively this role has to implement a publisher/subscriber mechanism. An agent that aims at providing knowledge (including trade with costs) will tell other agents, particularly esteemed brokers, about the kind of knowledge it is willing to offer. When asked for knowledge the provider weighs up whether or not to consent to the transfer depending on the importance and potential confidentiality of the requested knowledge and the social (e.g., organizational) relationship to the asking knowledge consumer.

Knowledge Broker: The knowledge broker acts as a yellow pages service within the system. It collects meta-knowledge on KM services (e.g., providers, other brokers, and processing services) and points a knowledgeseeking consumer to the right service. The broker also maintains a reputation list. Therefore it can rule out answers from unreliable partners upon request (Quality of Service enforcement). A broker may also act as a coordinator for adequate knowledge distribution within a legal organization or any other group of cooperating agents.

Knowledge Processing: This role provides services that generate or reveal new knowledge based on knowledge already available. This comprises semantic mediation and integration, learning, and inference which may be regarded as sub-roles, respectively. Inference is the KM function that reveals knowledge as a conclusion by logical deduction. Learning analyzes the knowledge base for generalization rules that may, e.g., allow deletion of inferable knowledge or prediction of recurring situations. The mediation function translates and possibly integrates knowledge from different sources and ontologies. In general, this service will be used within an agent by request of the consumer role or other sub-systems. In some cases it may also be offered as a service to other agents. Knowledge processing is the most complex role and demands for sophisticated reasoning and learning capabilities. Thus, only some agents will implement this role entirely.

Knowledge Acquisition: This internal role is intended to provide an interface to external data sources including sensors. Therefore it needs the capability to query a specific source and build up an internal representation 
of it. Changes in the source might trigger the generation of new knowledge items.

Knowledge Maintenance: This internal role incorporates tasks needed to keep the knowledge base manageable and to monitor changes. If required the role informs an agent's sub-systems of relevant changes which may trigger an update of situation assessment and planning.

Knowledge Output: An internal role providing an interface to the external environment through signal generators and user interfaces. The communication for this role is unidirectional toward the external interface. Possible responses from the environment are handled by knowledge acquisition which can of course be implemented within one agent.

It is important to reiterate that one instantiated agent can incorporate more than one role (e.g., an agent representing a truck can first act as a knowledge provider and later as a knowledge consumer). Hence, the incorporation of roles is a decomposition of the KM problem, which is in essence orthogonal to the mapping of organizational entities to agents. Furthermore, since different roles of agents need different reasoning capabilities, the encapsulation of roles can reduce the complexity of tasks which have to be performed by an agent at any given time.

\section{Consumer Provider Interaction}

A minimal role interaction model requires one agent $A$ in the knowledge consumer role and another agent $B$ in the knowledge provider role. In this section, we will discuss in more detail under which circumstances an agent assumes the role of a knowledge consumer and provider, respectively. We will describe how different parameter settings determine the decisions and actions associated with these roles.

The parameters are involved in the knowledge transfer process between consumer and provider. Adequate providers are identified by the consumer agent itself or by asking broker agents. Agent interactions in the transfer process may be modeled as an (iterated) contract net protocol with the consumer as initiator and at least one participating provider.

Consumer Parameters In general, the role of a knowledge consumer presupposes a situation which meets the conditions listed below:

1. The agent $A$ intends to obtain a knowledge item $k$ specified by a knowledge item description $d$. A knowledge item can be thought of as the truth value of a statement or the value of a variable. Its description $d$ can, in principle, be provided by a query, possibly in combination with additional constraints, e.g., the definition of a minimum precision for the intended response and a response deadline. These 
additional constraints make a knowledge item description different from a query or a sentence form which subsumes the intended knowledge item.

2. The knowledge item described by $d$ is not already part of $A$ 's knowledge base.

3 . The knowledge item described by $d$ cannot be inferred from $A$ 's knowledge base, given $A$ 's inferential capabilities.

4. $A$ believes that the knowledge item described by $d$ is, in principle, available now or later.

These prerequisites closely resemble the Gricean maxims on rational cooperative discourse (Grice 1975) in many respects. They have to be combined with additional criteria, e.g., the (estimated) cost of obtaining $k$, in order to cover situations where knowledge is a tradable good and the knowledge consumer has a limited budget for acquiring knowledge from other agents. In its interaction with other agents an agent in the knowledge consumer role has to make many decisions, including the following:

- It has to assign a rank or weight to $k$ in comparison to other knowledge items. This weight depends on how important $k$ is for achieving its current goals, and if there are alternative knowledge items which might serve the same or very similar purposes.

- The knowledge consumer has to choose among different knowledge sources, e.g., its sensors and knowledge provided by other agents. This decision can make use of the agent's own experiences from earlier knowledge transfers, or it can be made solely on the basis of a general trust/reputation mechanism.

- The agent has to decide upon the maximum acceptable price being paid for $k$ and the required response time.

- After each finished knowledge transfer, $A$ has to assess its quality, e.g., if the actually delivered knowledge item deviated significantly from what the agent expected in advance.

- Finally, the agent has to decide upon the next steps, e.g., if $k$ implies that other knowledge transfers are necessary.

These decisions of an agent assuming the knowledge consumer role are governed by the parameters importance, confidence, cost, availability, compliance, and value which are now discussed in more detail.

The importance parameter gives the (subjective) importance of a knowledge item $k$. The range of this parameter is between 0 (i.e., completely irrelevant) and 1 (maximum importance). As most other parameters, too, importance depends on the agent in question and time. Hence, we write $\operatorname{Imp}(A, k, t)$ for the importance an agent $A$ assigns to a knowledge item $k$ at 
time $t$. The importance parameter thus reflects an agent's point of view at a particular time which may differ significantly from the 'true' importance. The process of determining the importance of a knowledge item can be based on the agent's planning or risk management component (cf. (Lorenz e al. 2005) for details).

$\operatorname{Conf}(A, B, d, t)$ describes the confidence of the knowledge-consuming agent $\mathrm{A}$ at time $t$ that knowledge-providing agent $B$ will answer the knowledge request $d$ correctly. The parameter value ranges from -1 to 1 . -1 means $A$ feels certain that $B$ is lying or just has incorrect beliefs, whereas a confidence of 1 corresponds to absolute confidence in $B$ 's answer. 0 stands for neutral confidence, i.e., agent $A$ has no clue whether $B$ 's answer will be rather right or wrong. The parameter determines provider selection.

The cost parameter determines the maximum cost an agent is able and willing to accept for obtaining a knowledge item. Since cost, again, depends on an agent and time, we write $\operatorname{Cost}(A, k, t)$. This parameter includes costs arising in the communication process and possible costs to obtain $k$ as payment to the knowledge provider or knowledge brokers. The maximum accepted costs are closely related to $k$ 's importance and the agent's budget. In general, the accepted costs do not correspond to the price actually communicated to the provider.

A successful knowledge transfer presupposes that the knowledge item intended by the knowledge consumer agent is available, in principle. Hence, it is required that an agent assumes that there is a non-zero probability to obtain the intended item. This probability is given by the availability parameter. A zero availability, $\operatorname{Avail}(A, k, t)=0$, means that the agent does not believe that there is any chance to obtain $k$ at time $t$ and, hence, will not make any further attempts into that direction.

Availability of knowledge is based on prior experiences and background knowledge. It is used, for example, in deciding which knowledge items should be acquired (in case there are choices).

$\operatorname{Compl}\left(k^{\prime}, d\right)$ denotes the degree of compliance of the obtained knowledge item $k$ ' with respect to the intended knowledge specified by $d$. The value ranges from 0 to 1 . If the value is 0 the whole knowledge transfer has to be reiterated with another provider. The value 1 means that $k^{\prime}$ perfectly matches $d$. Item $k$ ' may differ in terms of spatial and temporal validity or precision of measurement. The consumer needs to evaluate kind and scale of a potential deviation in order to plan and execute appropriate actions to finally get the knowledge needed.

After a completed knowledge transfer, an agent determines how successful the transfer has been, i.e., its (net) value. This parameter depends on the initial importance of (the higher the initial importance, the higher its value), the compliance of the actually obtained knowledge item with the 
intended one (divergence decreases the value), and cost (the higher the cost the lower the value). The value of a knowledge transfer will affect the future behavior of an agent. Successful knowledge transactions with a particular provider agent, for example, will strengthen the connection between the involved agents and increase the likelihood of future transactions between them.

Provider Parameters Similar to the knowledge consumer role, the role of a knowledge provider incorporates multiple decisions during the transfer process which are characterized by a set of decision parameters to achieve a rational behavior. These parameters may be engaged in decisions that determine whether the provider is willing to transfer knowledge at all, or which transfer conditions the provider will propose and accept.

The proposed distributed knowledge management framework also considers agents being in competition or just belonging to different organizations. Thus, a provider agent needs to deliberate whether requested information may be propagated to some other agent or not. This confidentiality parameter is either defined by an explicit, predefined classification of the requested type or item of knowledge or may be determined by an intelligent estimation of the possible impact this knowledge may have on the providing agent and its organization if once revealed. Confidentiality is always specified with respect to an (agent) group of interest.

If the requested knowledge item is classified the provider agent will refuse to perform the answer action, except the asking agent has a sufficient security clearance which is organization-dependent. If the requested knowledge is considered basically confidential to some minor extent the provider's willingness to answer a given query strongly depends on the asking agent. This decision is influenced by the trust parameter. This parameter ranges from -1 to 1 and describes whether the consumer agent is supposed to comply with a non-disclosure agreement for the requested knowledge item. A trust value of 0 is neutral, i.e., the provider does not know anything about the consumer's trustworthiness.

Irrespective of confidentiality, trust, and transfer cost, an agent may have the disposition to agree to a knowledge transfer due to a social relation to the consumer agent. In this case, a transfer is motivated by a common organizational background, a current or past cooperation, or the aim to initiate a new (long-term) cooperation and to increase mutual trust (cf. (Alam et al. 2005)). The affinity parameter describes this disposition w.r.t. a specific agent and time. The parameter ranges from 0 (minimum affinity) to 1 (maximum). A high affinity decreases the minimum accepted price. If affinity is 1 the price is 0 , i.e., the knowledge is provided as a gift. 
In order to meet the consumer agent's requirements on precision and certainty the provider has to compute $\operatorname{Comp}\left(k^{\prime}, d\right)$. Due to different knowledge and/or inferential capabilities the value computed by the provider is not necessarily identical to the compliance value computed by the consumer agent after the completed knowledge transfer.

Whether the provider agrees to a knowledge transfer and under which conditions (minimum accepted price, response time) also depends on the expected expenses arising due to the transfer. This is represented by the provider's cost parameter. It may include a temporal and financial dimension consisting of, e.g., communication costs and reasoning costs.

If the provider is in general willing to answer an agent's query it determines the minimum accepted price for this service. The price is determined by the common value (if any), the provider's private value, the expected transfer cost, and the affinity to the consumer agent. The minimum accepted price, of course, may and in general will differ from the communicated price.

Interaction Protocol The knowledge transfer process between knowledge consumer and provider incorporates an informative act. Unfortunately, the FIPA Query Interaction Protocol is not sufficient to model all aspects of the depicted transfer process. As described above, the consumer request is more than just a query but a more complex description of the needed knowledge with constraints on that knowledge (e.g., precision) and the corresponding transfer process (e.g., response deadline and payment). Furthermore, the process may include a negotiation. Thus, we think of the actual knowledge transfer as a special action. In a consumer-initiated knowledge transfer process, the action with the preceding consumer's call for proposals, the provider's proposals, and possible negotiations are part of an (iterated) contract net protocol. The corresponding FIPA Interaction Protocol was adapted or interpreted for this special purpose (see Fig. 1.3). A provider-initiated process would follow the FIPA Propose Interaction Protocol. 


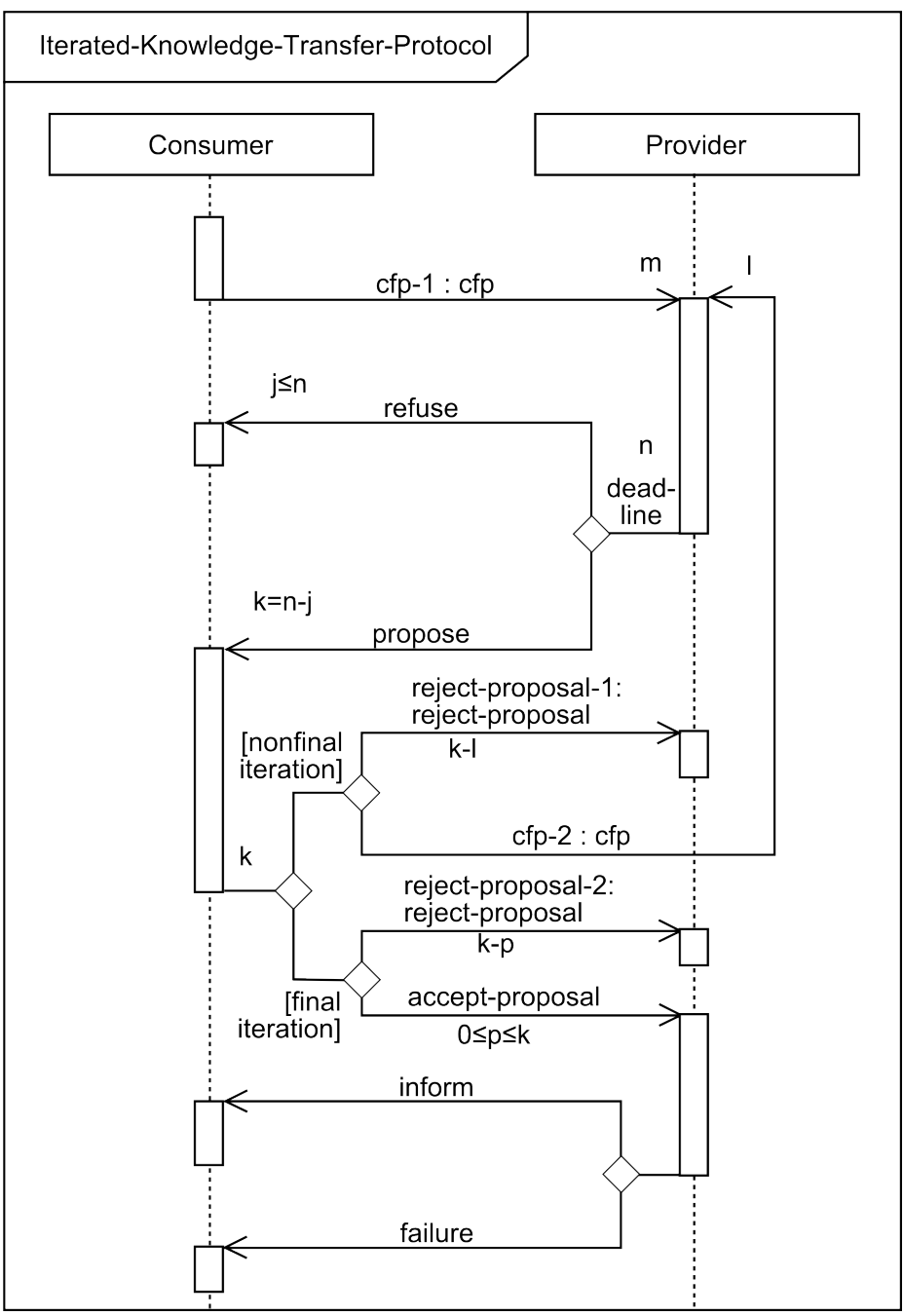

Fig. 1.3 Knowledge Transfer Interaction Protocol

\subsubsection{Conclusions}

We presented a framework for distributed knowledge management for modeling complex and dynamic scenarios from the logistics domain on the basis of multiagent systems. We introduced a set of knowledge management roles, decision parameters for them, and an interaction protocol for 
the two most important roles of our framework, the knowledge consumer and the knowledge provider.

This knowledge management framework is part of an agent-based logistic simulation system (Becker et al. 2006) and forms, together with a risk management component (Lorenz et al. 2005; Bemeleit et al. 2006), a dynamic knowledge-based decision system. This KM infrastructure offers the opportunity to simulate logistic processes as a combination of primary logistic tasks (e.g., transportation), knowledge processing/transfer, and decision-making.

\section{References}

Alam SJ, Hillebrandt F, Schillo M (2005) Sociological implications of gift exchange in multiagent systems. Journal of Artificial Societies and Social Simulation 8(3)

Becker M, Wenning BL, Goerg C, Gehrke JD, Lorenz M, Herzog O (2006) Agent-based and Discrete Event Simulation of Autonomous Logistic Processes. In: W. Borutzky and A. Orsoni and R. Zobel (eds) Proceedings of the 20th European Conference on Modelling and Simulation, Bonn, Sankt Augustin, Germany, pp. 566-571

Bemeleit B, Herzog O, Lorenz M, Schumacher J (2006) Proactive KnowledgeBased Risk Management. In: this volume, Chap 10

Bos A, de Weerdt MM, Witteveen C, Tonino J, Valk JM (1999) A dynamic systems framework for multi-agent experiments. In: European Summer School on Logic, Language, and Information. Foundations and Applications of Collective Agent Based Systems workshop

Bratman ME (1987) Intentions, Plans, and Practical Reason. Harvard University Press, Cambridge, MA

van Elst L, Dignum V, Abecker A (eds) (2004a) Agent-Mediated Knowledge Management: International Symposium AMKM 2003, Stanford, CA, USA, March 24-26, Revised and Invited Papers, LNCS 2926. Springer-Verlag, Berlin

van Elst L, Dignum V, Abecker A (2004b) Towards agent-mediated knowledge management. In: van Elst L et al. (eds) (2004), pp 1-30

Grice HP (1975) Logic and conversation. In: Cole P, Morgan JL (eds) Syntax and Semantics, vol 3, Speech Acts. Academic Press, New York, pp 41-58

Herrmann T, Jahnke I, Loser KU (2004) The role concept as a basis for designing community systems. In: Darses F, Dieng R, Simone C, Zacklad M (eds) COOP. IOS Press, pp 163-178

Hofmann O, Deschner D, Reinheimer S, Bodendorf F (1999) Agent-supported information retrieval in the logistics chain. In: HICSS '99: Proceedings of the Thirty-second Annual Hawaii International Conference on System Sciences, vol 8, IEEE Computer Society, p 8028 
Hult GTM, Cavusgiland ST, Calantone RJ (2003) Knowledge as a strategic resource in logistics and purchasing. Tech. Rep. Working Paper Series, No. 03001, Marketing Science Institute (MSI), Cambridge, Massachusetts

d'Inverno M, Luck M, Georgeff MP, Kinny D, Wooldridge M (2004) The dMARS architecture: A specification of the distributed multi-agent reasoning system. Autonomous Agents and Multi-Agent Systems 9(1-2):5-53

Jennings NR (2000) On agent-based software engineering. Artificial Intelligence 177(2):277-296

Lorenz M, Gehrke JD, Hammer J, Langer H, Timm IJ (2005) Knowledge management to support situation-aware risk management in autonomous, self-managing agents. In: Czap H, Unland R, Branki C, Tianfield H (eds) Self-Organization and Autonomic Informatics (I), Frontiers in Artificial Intelligence and Applications, vol 135. IOS Press, pp 114-128

Maurer M (2003) Important aspects of knowledge management. In: Klein R, Six HW, Wegner L (eds) Computer Science in Perspective: Essays Dedicated to Thomas Ottmann, LNCS 2598. Springer-Verlag, Berlin, pp 245-254

Rao AS, Georgeff MP (1991) Modeling rational agents within a BDI-architecture. Tech. Rep. 14, Australian AI Institute, Carlton, Australia

Scholz T, Timm IJ, Woelk PO (2004) Emerging capabilities in intelligent agents for flexible production control. In: Katalinic B (ed) Proceedings of the International Workshop on Emergent Synthesis (IWES 2004)

Smirnov A, Pashkin M, Chilov N, Levashova T (2003) Multi-agent knowledge logistics system "KSNet": Implementation and case study for coalition operations. In: Mař́k V et al. (eds) CEEMAS 2003, LNAI 2691, pp 292-302

Timm IJ (2004) Dynamisches Konfliktmanagement als Verhaltenssteuerung Intelligenter Agenten. Dissertationen in Künstlicher Intelligenz, vol 283, Akademische Verlagsgesellschaft, Berlin

Wooldridge M, Lomuscio A (2001) A computationally grounded logic of visibility, perception and knowledge. Logic Journal of the IGPL 9(2):257-272

Zice S, Zhengping L, Runtao Q, Mansoor S (2001) Agent-based logistics coordination and collaboration. Tech. Rep. SIMTech (AT/01/011/LCI), Singapore Institute of Manufacturing Technology 